\title{
Le controlling dans la planification directrice cantonale
}

\section{Marco Keiner, Nicolas Mettan, Barbara Schultz, Zurich et Lausanne}

\section{La gestion actuelle du plan directeur cantonal est déficiente}

Dans la pratique actuelle de la planification directrice, un remaniement intégral du plan directeur a lieu en règle générale tous les dix ans. Le plan directeur reste le plus souvent inchangé durant toute sa durée de validité, et sa gestion se fait de manière peu systématique. Jusqu'à la prochaine évaluation, qui est généralement faite peu de temps avant la révision complète, l'efficacité du plan directeur ne peut être appréciée que de façon sommaire. Une vue d'ensemble des évolutions souhaitées et non souhaitées de même que des effets entrainés par les mesures du plan directeur fait habituellement défaut, de sorte qu'il n'est pas possible d'intervenir pour réorienter et corriger des tendances erronées. Ce problème est connu depuis des années (Conférence des offices romands d'aménagement du territoire (CORAT) 1993, Egli, Ringli \& SchmidT 1995) mais n'a pas pu être résolu de manière satisfaisante à ce jour. Le plan directeur souffre donc encore d'un manque d'efficacité.

La question de la dynamique d'un plan directeur cantonal redevient importante eu égard à la stratégie poursuivie par l'Office fédéral du développement territorial (ODT) d'orienter l'aménagement du territoire vers le principe de la durabilité.

Or, un développement spatial axé sur le principe de la durabilité nécessite précisément que des moyens de régulation soient mis en œuvre. Il s'agit de prendre les dispositions qui s'imposent pour pouvoir identifier les écarts par rapport au cours que doit suivre l'aménagement du territoire et procéder à d'éventuelles corrections.

Il existe des instruments capables de contrôler et d'orienter la mise en œuvre des objectifs et des mesures découlant des plans directeurs cantonaux. Ces instruments peuvent aussi contribuer à vérifier si les développements souhaités ont bien eu lieu. Des évolutions spatiales non durables peuvent ainsi être décelées à un stade précoce et corrigées à l'aide de mesures adéquates. Ces instruments sont le monitoring, le controlling et le benchmarking (appuyés par des indicateurs). Un développement spatial durable (et une planification directrice continue) n'est guère envisageable sans le recours à une telle instrumentation (KEINER, Schultz \& SCHMID 2001).
L'instrument central de ce système, le controlling, est présenté ci-après et son utilité pour la planification directrice cantonale est discutée. Prochainement, un outil de travail sur l'intégration de la problématique du développement durable dans la planification directrice sera mis à la disposition des cantons (INFRAS/ ORL/C.E.A.T. 2001).

\section{Définition du controlling}

Le controlling est une notion relativement floue. Dans la littérature anglo-américaine sur le management, la fonction de "controlling» est présentée comme l'une des composantes du processus de gestion, à côté d'autres notions telles que "planning», «coordinating», «organizing» ou «directing». Aux Etats-Unis, le concept du controlling existe depuis les années 30. Il tire ses origines non pas de la théorie de l'économie d'entreprise mais de la pratique entrepreneuriale. En Europe occidentale, le controlling ne s'est imposé que vers les années 70 . Il est aujourd'hui compris comme instrument d'une conduite d'entreprise globale, prospective et axée sur les objectifs dans le circuit des dispositifs de planification, de mise en œuvre, de contrôle et de (ré-)orientation. Il constitue ainsi le pilier d'un système supérieur de gestion d'entreprise comprenant par ailleurs le système d'organisation, la gestion du personnel et la conception directrice de l'entreprise. Le controlling se compose des objectifs poursuivis par l'entreprise et des mesures prévues pour y parvenir. Des comparaisons constantes entre ce qui doit être et ce qui est (objectifs/réalité) permet à la direction de l'entreprise de savoir si les objectifs établis ont été atteints. Une analyse continue et étayée de tout éloignement des objectifs dans un système de «reporting» détermine les mesures à prendre pour réorienter le cours des choses. Il paraît déjà clair à ce stade que «controlling» n'est pas tout à fait synonyme de «contrôle» mais qu'il correspond plutôt au sens de «to control» (= conduire, réguler).

Abrégé apostillé au préalable de l'étude «Planification directrice cantonale et développement durable», élaborée par INFRAS/Institut pour l'aménagement national, régional et local (ORL)/Communauté d'études pour l'aménagement du territoire (C.E.A.T.); autorisé par l'Office fédéral du développement territorial (ODT). 


\section{Niveaux de controlling}

\section{Niveau stratégique (principes directeurs)}

Contrôle de réalisation des objectifs

$\longleftarrow \quad$ Indicateurs principaux

Contrôle de validité des objectifs

Processus participatif

\section{Niveau opérationnel (mesures visant à assurer la coordination)}

Contrôle d'exécution

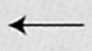

Listes de contrôle

Contrôle des effets

$\longleftarrow$ Indicateurs

Fig. 1: Les différents niveaux de controlling dans la planification directrice

The different levels of controlling in directive planning

Die einzelnen Ebenen des Richtplan-Controlling

\section{Le controlling permet une planification directrice continue et axée sur les résultats}

Le controlling dans la planification directrice est un instrument de conduite pour l'adaptation continue du plan directeur. L'état des lieux mis en évidence par les contrôles de réalisation des objectifs, de l'exécution et des effets est comparé aux objectifs visés et au plan d'action (état souhaité). Au besoin, des mesures sont définies pour parer à des développements spatiaux non souhaités. Le Guide «Le plan directeur cantonal» (Office fédéral de l'aménagement du territoire - OFAT 1997) considérait déjà le controlling comme «composante de la planification directrice». Pour un plan directeur cantonal, conçu comme un instrument de conduite moderne dont l'efficacité doit pouvoir être mesurée, la procédure de controlling constitue un mécanisme de contrôle qui accompagne tout le processus.

En avertissant de la nécessité d'intervenir et d'adapter le plan directeur, le controlling contribue à dynamiser la planification directrice. Son efficacité s'en trouve renforcée, ce qui répond au principe d'un développement durable (Communauté d'études pour l'aménagement du territoire - C.E.A.T. 2000). Pour qu'il ait caractère contraignant pour les autorités, le controlling du plan directeur devrait être intégré dans le plan directeur lui-même. Sous forme de mesure visant à assurer la coordination, le controlling devrait devenir une tâche permanente de la planification directrice dont la procédure, les compétences et la périodicité sont fixées de manière précise.

Le controlling du plan directeur n'est pas séparé des autres plans et instruments de coordination du canton. Il doit donc s'insérer sans contradiction dans un système global supérieur d'évaluation de la politique cantonale. S'il existe déjà un système de controlling d'ordre supérieur au sein d'une administration cantonale ayant introduit des réformes de type NGP (Nouvelle gestion publique), le controlling du plan directeur doit y être adapté et doit surtout préciser - sur la base du plan directeur cantonal - les effets qu'ont les interventions de l'Etat sur l'organisation du territoire. Le controlling du plan directeur devrait également être compatible avec des démarches du même genre existant déjà dans d'autres cantons et à l'échelle de la Confédération.

\section{Le controlling du plan directeur a lieu à différents niveaux}

En principe, le controlling fait une distinction entre le niveau stratégique et le niveau opérationnel. Au niveau stratégique, les objectifs (= les principes directeurs) sont soumis à un contrôle des objectifs et, au niveau opérationnel, les mesures du plan directeur (= les indications visant à assurer la coordination) sont soumises à un contrôle d'exécution et à un contrôle des effets (GatTi-Sauter 1995) (cf. figure 1).

\subsection{Niveau stratégique}

Le contrôle des objectifs comprend deux aspects:

Contrôle de réalisation des objectifs: En comparant ce qui doit être (principes directeurs) et ce qui est (développement spatial effectif), on peut constater si les objectifs de la planification directrice ont été atteints. Cela ne peut se faire que si les principes directeurs ont été formulés de façon concrète et selon un ordre de priorité. L'atteinte des buts peut être évaluée au moyen d'indicateurs pertinents. Dans le cadre d'un concept de controlling, ces indicateurs dits principaux peuvent être choisis déjà au moment de la définition des principes directeurs.

Contrôle de validité des objectifs: Il s'agit aussi d'examiner si, du point de vue de l'efficacité de la planifi- 
cation directrice et en fonction des nouvelles tendances du développement spatial, les principes directeurs eux-mêmes sont toujours opportuns et appropriés ou s'ils doivent être révisés, respectivement si les principes directeurs reflètent bien les principaux postulats en matière d'aménagement du territoire. Ce processus peut être conçu de manière participative, en s'appuyant sur les résultats du monitoring et du controlling ainsi que sur d'autres études de base.

Cas type: Programme de controlling du Canton de Lucerne

Le canton de Lucerne est pionnier dans ce domaine: en 1998 déjà, il a développé un concept (interne) de controlling pour le plan directeur. Celui-ci est notamment basé sur l'étude commandée en 1995 par la COSAC: «Exigences en matière de contrôle et de mise à jour d'un plan directeur cantonal».

Ce concept est aujourd'hui sur le point d'être terminé avec le soutien de la division de planification du paysage et de l'environnement (LEP) de l'institut ORL à l'EPFZ. Un intérêt primordial est accordé à l'élaboration des indicateurs de controlling du plan directeur cantonal (PDC) au niveau stratégique. Ces indicateurs sont basés sur le jeu d'indicateurs de l'outil de travail (INFRAS/ORL/ C.E.A.T. 2001), ce qui permet une comparaison avec d'autres cantons (benchmarking), et spécifiés selon les principes directeurs du PDC du canton de Lucerne.

\subsection{Niveau opérationnel}

Contrôle d'exécution: Ce contrôle consiste à vérifier si les mesures visant à assurer la coordination et destinées à atteindre les objectifs stratégiques ont été exécutées et quelles ont été les ressources utilisées à cet effet. On peut envisager pour cela de mettre à jour les différentes fiches d'objet, respectivement de coordination, du plan directeur au moyen de listes de contrôle dans une application de la banque de données (état d'avancement de la planification: information préalable - coordination en cours - coordination réglée).

Contrôle des effets: L'évaluation des mesures de coordination a pour but de savoir si leur mise en œuvre a déployé des effets et si ces effets vont dans le sens voulu. Une série d'indicateurs permet d'établir si et dans quelle mesure elles ont contribué à atteindre les objectifs fixés par les principes directeurs. Si le contrôle des effets révèle que les mesures de coordination n'ont pas entraîné les effets escomptés, il convient de les adapter. Il serait assurément trop long et coûteux de vouloir examiner toutes les mesures d'aménagement d'un plan directeur à l'aide d'indicateurs. Les mesures visant à assurer la coordination devraient donc être hiérarchisées. Et pour celles considérées comme prioritaires, il conviendrait de définir des indicateurs lors de la mise au point du concept de controlling.

Cas type: Contrôle d'exécution des mesures du plan directeur du Canton du Valais visant à assurer la coordination

Pour la gestion et la mise en œuvre de son plan directeur, le canton du Valais a adopté une méthode de planification continue. Grâce à cette démarche, le Valais a pu faire l'économie d'un remaniement intégral du plan directeur, comme le prévoit la loi sur l'aménagement du territoire.

Une analyse de l'évolution et de la situation de l'aménagement du territoire dans le canton est effectuée au début de chaque année en vue d'orienter la planification cantonale et d'établir le programme de travail pour l'année à venir. Un bilan des modifications du plan directeur intervenues au cours de l'année écoulée est également effectué.

C'est ainsi que depuis l'adoption du premier plan directeur en 1988, une trentaine d'études d'approfondissement ont été menées et que 66 fiches de coordination ont été révisées et transmises à l'autorité fédérale pour approbation. Chaque année, toutes les instances concernées par la planification directrice reçoivent un exemplaire des fiches de coordination modifiées et sont priées de les insérer dans le classeur du plan directeur, permettant à celui-ci d'être constamment à jour.

\section{Indicateurs pour le controlling du plan directeur}

Un indicateur est une unité de mesure ou d'observation qui décrit un état de faits ne pouvant pas être appréhendé directement. L'intérêt réel ne porte pas sur l'indicateur lui-même mais sur l'état indiqué des faits. Il doit donc exister une relation causale entre le fait indiqué et l'indicateur. L'utilité des indicateurs dépend avant tout de leur aptitude à reflèter des états de fait avec justesse (Blanchet \& November 1998).

Les indicateurs sont là pour produire des informations facilement compréhensibles et mesurables sur le développement spatial. Ils servent à créer les bases d'une évaluation objective des choses et contribuent de ce fait à l'objectivation des processus de décision et au contrôle de l'efficacité des mesures. Ces fonctions des indicateurs sont les mêmes dans la planification directrice, et c'est pourquoi ils constituent une assise indispensable tant du monitoring et du controlling que du benchmarking (Keiner, Schultz \& Schmid 2001) (cf. tableau 1). 


\begin{tabular}{|c|c|c|}
\hline $\begin{array}{c}\text { Objectifs du } \\
\text { développement durable } \\
\text { appliqués à } \\
\text { l'aménagement du } \\
\text { territoire }\end{array}$ & Indicateur principal & Valeur-cible \\
\hline \multirow[t]{5}{*}{$\begin{array}{l}\text { Densifier le milieu bâti } \\
\text { et utiliser le sol avec } \\
\text { parcimonie }\end{array}$} & $\begin{array}{l}\text { Dimension des zones à bâtir déjà } \\
\text { construites }\end{array}$ & $\begin{array}{l}\qquad= \\
\text { resp. augmentation aussi } \\
\text { faible que possible }\end{array}$ \\
\hline & $\begin{array}{l}\text { Dimension des zones à bâtir non } \\
\text { construites (réserves de zones à } \\
\text { bâtir) }\end{array}$ & - \\
\hline & $\begin{array}{l}\text { Taux d'utilisation: rapport entre la } \\
\text { surface brute de plancher construite } \\
\text { et la totalité de la surface brute de } \\
\text { plancher légalement admise }\end{array}$ & + \\
\hline & $\begin{array}{l}\text { Proportion des surfaces urbanisées } \\
\text { et de transport par rapport à la } \\
\text { superficie totale du canton }\end{array}$ & $=$ \\
\hline & $\begin{array}{l}\text { Surface de zone à bâtir par habitant, } \\
\text { resp. par personne active }\end{array}$ & - \\
\hline \multirow{3}{*}{$\begin{array}{l}\text { Réduire le trafic en } \\
\text { harmonisant le déve- } \\
\text { loppement de l'urba- } \\
\text { nisation et les trans- } \\
\text { ports publics et pro- } \\
\text { mouvoir un tourisme } \\
\text { compatible avec } \\
\text { l'environnement }\end{array}$} & $\begin{array}{l}\text { Répartition modale: prestation de } \\
\text { transport en personnes-kilomètres } \\
\text { par mode de transport et but de } \\
\text { déplacement }\end{array}$ & $\begin{array}{l}\text { Réduire la part du trafic } \\
\text { motorisé individuel }\end{array}$ \\
\hline & $\begin{array}{l}\text { Part de la population desservie par } \\
\text { les transports publics } \\
\text { (périmètres: train } 500 \mathrm{~m} \text {, } \\
\text { bus/tramway } 250 \mathrm{~m} \text {; cadence: } \\
\text { minimum } 30 \text { minutes) }\end{array}$ & + \\
\hline & $\begin{array}{l}\text { Emissions de } \mathrm{CO}_{2} \text { par habitant et } \\
\text { par année }\end{array}$ & $\begin{array}{c}\text { Réduction de } 10 \% \text { entre } \\
2000 \text { et } 2010 \\
\text { (SuisseEnergie) }\end{array}$ \\
\hline \multirow{3}{*}{$\begin{array}{l}\text { Garantir la qualité de } \\
\text { l'environnement, pro- } \\
\text { téger la population des } \\
\text { dangers naturels ainsi } \\
\text { que des nuisances et } \\
\text { des risques produits } \\
\text { par le traitement des } \\
\text { déchets et les } \\
\text { décharges }\end{array}$} & $\begin{array}{l}\text { Part de la population exposée à des } \\
\text { nuisances causées par le bruit, } \\
\text { supérieures à la valeur limite } \\
\text { d'immission }(60 \mathrm{~dB}(\mathrm{~A}))\end{array}$ & $\begin{array}{l}\text { - } \\
\text { Respect des valeurs limites } \\
\text { d'émission selon } \\
\text { l'ordonnance sur la } \\
\text { protection contre le bruit } \\
\text { (OPB) }\end{array}$ \\
\hline & $\begin{array}{l}\text { Qualité de l'air dans les zones urba- } \\
\text { nisées }\end{array}$ & $\begin{array}{l}+ \\
\text { Respect des valeurs limites } \\
\text { d'émission selon } \\
\text { l'ordonnance sur la } \\
\text { protection de l'air (OPair) }\end{array}$ \\
\hline & $\begin{array}{l}\text { Proportion de surfaces contaminées } \\
\text { déjà assainies et répertoriées par } \\
\text { rapport à la surface totale des sites } \\
\text { contaminés et potentiellement } \\
\text { contaminés, recensés par le cadastre }\end{array}$ & + \\
\hline
\end{tabular}

Tab. 1: Objectifs du développement durable appliqués à l'aménagement du territoire, indicateurs principaux pour le contrôle de réalisation des objectifs et valeurs-cibles (les indicateurs principaux traduisent l'évolution dans le temps).

Sustainable spatial development objectives, key indicators for strategic controlling and goals (the key indicators expressing an evolution in time). 


\begin{tabular}{|c|c|c|}
\hline & $\begin{array}{l}\text { Part de la population vivant dans les } \\
\text { zones de dangers (dangers naturels, } \\
\text { risques techniques) }\end{array}$ & - \\
\hline \multirow{3}{*}{$\begin{array}{l}\text { Mettre en réseau, } \\
\text { sauvegarder et valoriser } \\
\text { les paysages intacts et } \\
\text { proches de l'état nat urel }\end{array}$} & $\begin{array}{l}\text { Volume des bâtiments hors de la zone } \\
\text { à bâtir }\end{array}$ & $=$ ou - \\
\hline & $\begin{array}{l}\text { Proportion de surfaces paysagères non } \\
\text { morcelées }\end{array}$ & + \\
\hline & $\begin{array}{l}\text { Proportion de zones protégées par } \\
\text { rapport à la superficie totale }\end{array}$ & $=\mathrm{Ou}+$ \\
\hline \multirow{2}{*}{$\begin{array}{l}\text { Viser une décentrali- } \\
\text { sation concentrée des } \\
\text { activités écono miques, } \\
\text { des fonctions dévolues } \\
\text { aux centres et des pôles } \\
\text { urbains }\end{array}$} & $\begin{array}{l}\text { Part de la population pouvant attein- } \\
\text { dre le prochain centre régional par les } \\
\text { transports publics en moins de } 45 \\
\text { minutes }\end{array}$ & + \\
\hline & $\begin{array}{l}\text { Proportion de personnes actives dans } \\
\text { les pôles de développement par rap- } \\
\text { port au total de la population active } \\
\text { dans le canton }\end{array}$ & + \\
\hline $\begin{array}{l}\text { Utiliser l'énergie de } \\
\text { manière efficace et } \\
\text { rationnelle }\end{array}$ & $\begin{array}{l}\text { Consommation globale d'énergie par } \\
\text { groupe de consommateurs et par agent } \\
\text { énergétique }\end{array}$ & $\begin{array}{l}\text { - } \\
\text { Réduire la consommation } \\
\text { d'énergies fossiles de } 10 \% \\
\text { jusqu'en } 2010 \\
\text { (SuisseEnergie) }\end{array}$ \\
\hline $\begin{array}{l}\text { Garantir l'accès au } \\
\text { facteur de production sol } \\
\text { dans les pôles de } \\
\text { développement }\end{array}$ & $\begin{array}{l}\text { Part de surface brute de plancher } \\
\text { disponible pour l'industrie, le com- } \\
\text { merce et les services dans les pôles de } \\
\text { développement }\end{array}$ & $=\mathrm{ou}+$ \\
\hline \multirow{2}{*}{$\begin{array}{l}\text { Garantir l'accès aux } \\
\text { services de base dans les } \\
\text { domaines de l'énergie et } \\
\text { des télé communications }\end{array}$} & $\begin{array}{l}\text { Part de la surface d'habitat et d'infra- } \\
\text { structure avec raccordement au câble } \\
\text { et réception GSM (resp. UMTS) }\end{array}$ & + \\
\hline & $\begin{array}{l}\text { Evolution des prix (énergie, télécom- } \\
\text { munica tions) dans les régions péri- } \\
\text { phériques par rapport aux régions } \\
\text { urbaines }\end{array}$ & uniforme \\
\hline \multirow[t]{2}{*}{$\begin{array}{l}\text { Conserver et mettre en } \\
\text { valeur les paysages } \\
\text { ruraux par une exploi- } \\
\text { tation agricole naturelle }\end{array}$} & $\begin{array}{l}\text { Proportion de surface exploitée de } \\
\text { façon écologique par rapport au total } \\
\text { de la surface agricole }\end{array}$ & $\begin{array}{c}+ \\
20 \% \text { de la surface agricole } \\
\text { utile } \\
\text { (Paysage 2020) }\end{array}$ \\
\hline & Surface totale d'assolement & $\begin{array}{c}\text { selon les exigences posées par } \\
\text { le Plan sectoriel des surfaces } \\
\text { d'assolement (SDA) }\end{array}$ \\
\hline $\begin{array}{l}\text { Protéger les sites } \\
\text { construits et les paysages } \\
\text { typiques dignes d'être } \\
\text { conservés }\end{array}$ & $\begin{array}{l}\text { Nombre de sites naturels et construits } \\
\text { inventoriés }\end{array}$ & $=\mathrm{ou}+$ \\
\hline
\end{tabular}

Légende: + doit augmenter ; = doit rester identique ; - doit diminuer

Suite: Raumplanerische Nachhaltigkeitsziele, Leitindikatoren für das Zielerreichungscontrolling und Zielgrössen (die Leitindikatoren verstehen sich jeweils als Entwicklungsgrösse über die Zeit).

Source: INFRAS/Institut für Orts-, Regional- und Landesplanung ORL/Communauté d'études pour l'aménagement du territoire C.E.A.T. (2001) 
Les indicateurs ne peuvent toutefois pas mesurer tous les objectifs de la planification directrice et ne recouvrent qu'une partie du développement spatial effectif. En outre, maints indicateurs ne reflètent qu'indirectement la relation de causalité existant avec la planification directrice, car le plan directeur n'est qu'un élément parmi beaucoup d'autres qui déterminent le développement spatial. Il est parfois difficile d'établir avec certitude si c'est réellement l'action du plan directeur qui a fait évoluer les conditions spatiales dans le sens souhaité. En cela, il ne faut pas perdre de vue que c'est l'interprétation des indicateurs qui leur donne leur signifiance et que les données quantitatives doivent toujours être complétées par des appréciations qualitatives. Il conviendrait donc d'évaluer dans quelle mesure un développement donné a été influencé par l'application du plan directeur et dans quelle mesure ce développement est dû à d'autres facteurs d'influence.

\section{Réalisation du controlling du plan directeur}

L'élaboration d'un système de controlling pour la planification directrice étant une terra incognita pour la plupart des cantons, l'Office fédéral du développement territorial (ODT), en collaboration avec l'Office fédéral de l'environnement, des forêts et du paysage (OFEFP) et le Secrétariat d'Etat à l'économie (SECO) a chargé la division de planification du paysage et de l'environnement (LEP) de l'Institut pour l'aménagement national, régional et local (ORL) à l'EPFZ, la Communauté d'études pour l'aménagement du territoire (C.E.A.T.) et le bureau INFRAS d'élaborer un outil de travail. Ce document (INFRAS/ ORL/C.E.A.T. 2001) visant à aider les cantons à élaborer un système de controlling spécifique édité par l'Office fédéral du développement territorial (ODT), l'Office fédéral de l'environnement, des forêts et du paysage (OFEFP) et le Secrétariat d'Etat à l'économie (SECO). Au-delà, il sera judicieux d'instaurer des groupes de travail intercantonaux pour l'échange d'expériences, par exemple dans le cadre de la Conférence suisse des aménagistes cantonaux (COSAC), ou à l'échelle romande, dans le cadre de la Conférence des offices romands d'aménagement du territoire (CORAT). Une telle coopération permettrait de créer des synergies entre cantons aussi bien au stade de la conception qu'à celui de la mise en application ultérieure.

Un controlling du plan directeur devrait pouvoir s'effectuer à intervalles rapprochés mais aussi à des coûts supportables. A l'instar de l'observation «continue» du territoire, un controlling "axé sur le processus» ne signifie pas que quelqu'un doive s'en occuper en permanence tous les jours. Mais, d'autre part, le risque existe aussi que le controlling soit noyé par les affaires courantes. Pour toutes ces raisons, il serait judicieux de coupler le controlling à d'autres échéances. Le compte-rendu périodique à l'attention de l'autorité fédérale pourrait être l'occasion de communiquer tous les quatre ans les résultats du controlling au niveau stratégique (contrôle des objectifs - adaptation de principes directeurs).

En revanche, au niveau opérationnel, les intervalles de controlling devraient être plus courts (par ex. chaque deux ans), car les mesures visant à assurer la coordination exigent une mise à jour permanente pour maintenir le dynamisme de la planification directrice.

Un système possible de controlling et des suggestions pour une palette d'indicateurs principaux sont esquissés dans l'outil de travail (INFRAS/ORL/C.E.A.T. 2001) mentionné. Il servira de point de repère pour la réalisation du monitoring, du controlling et du benchmarking au niveau cantonal. Les indicateurs principaux sont des indicateurs qui couvrent un domaine relativement étendu et qui composent un ensemble cohérent. Ils doivent mesurer les modifications du développement spatial intervenues au cours d'une période donnée. Chaque indicateur principal est assorti d'une valeur-cible représentée essentiellement par son évolution souhaitable.

Les conditions topographiques et les structures démographiques et économiques étant différentes d'un canton à l'autre, les indicateurs principaux et leurs valeurs-cibles vont différer de canton en canton. Ainsi, l'organisation finale du controlling sera spécifique dans chaque canton et pourra être simplifiée en fonction des exigences respectives.

Cas type: Controlling du plan directeur du Canton de Berne

Le nouveau projet du plan directeur pour la participation (2001) prévoit d'examiner systématiquement le plan directeur quant à son efficacité à concrétiser les buts fixés. Une distinction est opérée entre les effets prévus et les prestations fixées. Un effet prévu suppose l'existence d'une ligne directrice définie sur le plan politique (niveau stratégique). Une prestation fixée se réfère à une prestation quantifiable devant être atteinte par l'administration (niveau opérationnel). Elle est déterminée en fonction de mesures opérationnelles.

Une fiche de contrôle est établie pour chaque effet prévu et pour chaque prestation fixée. Cette fiche, qui contient entre autres des indicateurs et des valeurs estimées pour l'atteinte des objectifs, est tenue à jour par le service chargé de l'application des mesures. 
Il est prévu de mettre sur pied un service de controlling qui surveillera la mise en œuvre des mesures dans les délais impartis. Celui-ci établira tous les deux ans un rapport de controlling à l'attention de l'autorité supérieure.

Le contrôle des effets relève également de la compétence du service de controlling. Ce dernier assemble à cette fin les données provenant du contrôle des prestations fixées et des résultats tirés de l'observation du territoire.

\section{Le benchmarking dans la planification directrice}

Un benchmarking étendu à la Suisse entière, mais qui ne doit pas être un concours de la «meilleure» planification directrice, serait un complément judicieux du controlling (KEINER, SCHULTz \& SCHMID 2001). Le concept du benchmarking a été introduit dans la gestion d'entreprise au début des années 80 . Il consiste à mesurer de façon continue des produits, des services et des pratiques à l'aune des entreprises les plus fortes qui dominent la branche. Le benchmarking implique aussi la recherche des «meilleures pratiques» conduisant à des prestations de pointe. Il s'agit non seulement de déceler les écarts de performance par rapport à d'autres entreprises mais aussi de trouver et d'assimiler les méthodes et les démarches permettant d'améliorer les prestations. Pour mettre en évidence les différences de performance, on peut se servir de quelques indicateurs principaux quantitatifs mais aussi de mesures qualitatives provenant de l'analyse des données et des informations des entreprises considérées. Avec l'adoption de méthodes inspirées de la nouvelle gestion publique, le benchmarking devient de plus en plus courant dans le secteur public également.

Les cantons auraient ainsi l'occasion de se positionner dans un cadre plus large et de voir comment et avec quel résultat des cantons comparables tentent d'orienter le développement spatial par le biais de leur plan directeur.

Le but d'un benchmarking de la planification directrice est d'accroître l'efficacité du produit "plan directeur», de mieux structurer le déroulement et l'organisation de la planification directrice cantonale et de montrer à quel stade sont parvenus les cantons sur la voie d'un développement spatial durable. Une comparaison avec d'autres cantons permettrait de tirer des enseignements de leurs «meilleures pratiques», de prendre conscience de ses propres déficits éventuels et d'en trouver les causes (MAIER, Weber \& Zuber 2000).

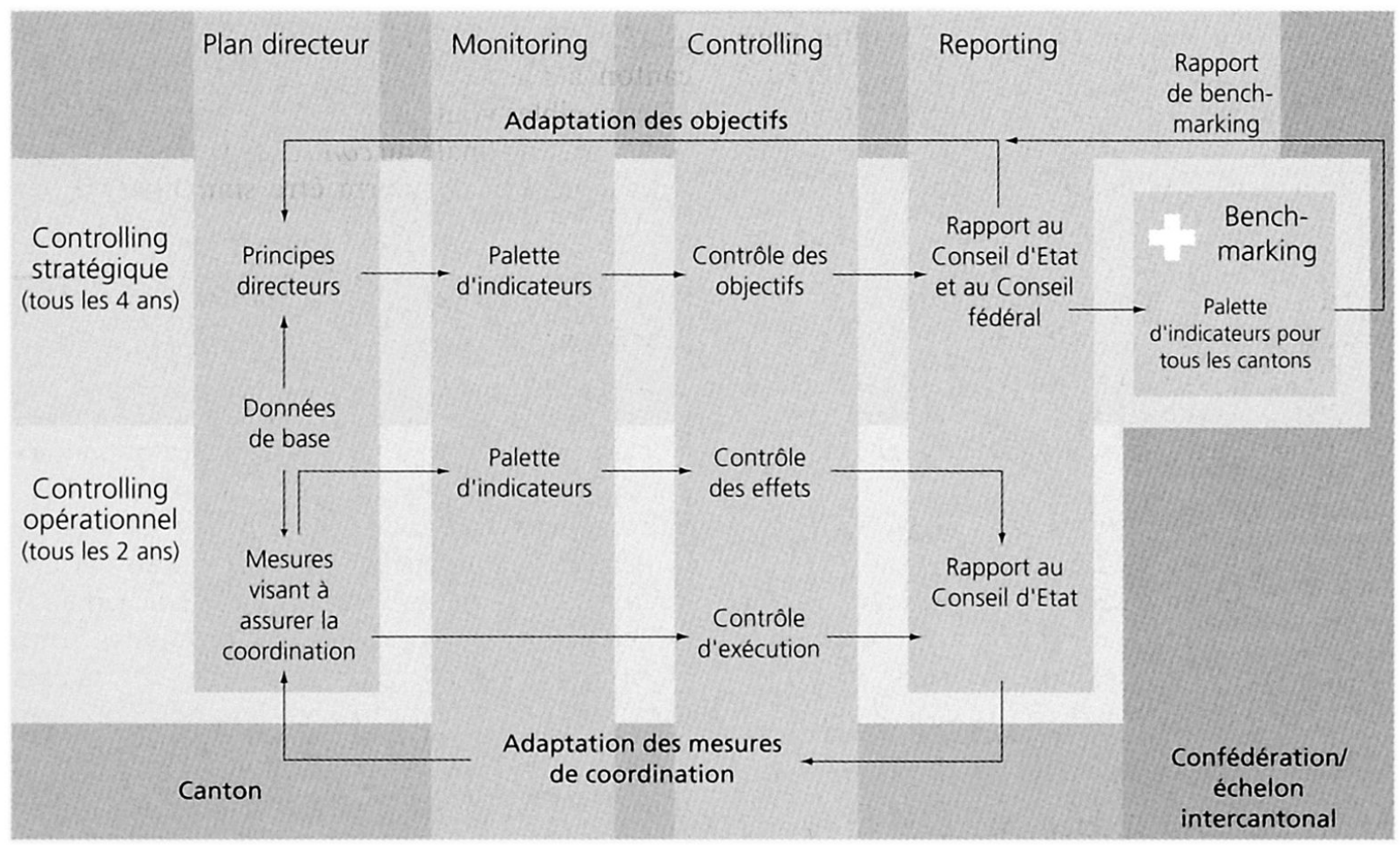

Fig. 2: Le système de monitoring, controlling et benchmarking dans la planification directrice cantonale The system of monitoring, controlling and benchmarking in cantonal directive planning Das System von Monitoring, Controlling und Benchmarking in der kantonalen Richtplanung 


\section{Vers un système intégré de monitoring, de controlling et de benchmarking de la planification directrice cantonale}

Le graphique (figure 2) illustre schématiquement une proposition de schéma intégré de monitoring, de controlling et de benchmarking avec ses effets sur la planification directrice cantonale.

Au niveau stratégique, les principes directeurs sont soumis à un monitoring à travers des indicateurs. En résulte des données et des informations qualitatives qui servent à un contrôle des objectifs. Ce contrôle des objectifs se fait tous les quatre ans et est documenté dans un rapport au Conseil d'Etat et au Conseil fédéral. Par la suite, les objectifs du plan directeur peuvent être adaptés, c'est-à-dire rectifiés selon le processus prévu par l'article 9 de la loi fédérale sur l'aménagement du territoire (LAT) et l'article 9 de l'ordonnance sur l'aménagement du territoire (OAT).

Les mesures visant à assurer la coordination sont à soumettre à un contrôle des effets, qui se fait sur la base d'une palette d'indicateurs, ainsi qu'à un contrôle d'exécution. Les résultats sont consignés dans un rapport annuel ou bis-annuel au Conseil d'Etat et permettent une adaptation des mesures de coordination du plan directeur.

Le benchmarking, en tant qu'instrument supplémentaire pour la comparaison du développement spatial cantonal sur le plan suisse, se base sur le controlling stratégique. Il sert comme point de repère et peut entraîner des adaptations ultérieures des objectifs.

\section{Références}

Blanchet, C. \& A. November (1998): Indicateurs du développement durable appliqués à l'aménagement du territoire. - Institut universitaire d'études du développement; CES, Genève.

C.E.A.T. (2000): Du concept de développement durable à sa mise en œuvre. - Lausanne.

Conférence des Offices Romands D'Aménagement Du Territoire (CORAT) (1993): Succès et lacunes de la planification cantonale. Bilan des plans directeurs cantonaux.

Egli, K., Ringli, H. \& U. Schmidt (1995): Auf dem Weg zu einer wirkungsvolleren kantonalen Richtplanung. Zwischenbericht 1995 aus einem ständigen Erfahrungsaustausch an der ETH Zürich. ORL-Bericht 95, Zürich. Gatti-Sauter, S. (1995): Anforderungen an Kontrollierbarkeit und Fortschreibung eines kantonalen Richtplans. Denkansätze und Diskussionsbasis. Bericht zuhanden der Schweizerischen Kantonsplaner-
Konferenz, Kommission Richtplanung, Arbeitsgruppe «Contrôle et Gestion», Neuhausen.

INFRAS/ORL/C.E.A.T. (2001): Planification directrice cantonale et développement durable. - Un outil de travail, Berne, édité par ODT/OFEFP/SECO.

Keiner, M., Schultz, B. \& W.A. Schmid (2001): Nachhaltige kantonale Richtplanung. - = DISP 146.

Maier, J., Weber, A. \& A. Zuber (2000): Benchmarking auch in der Regionalpolitik? Was kann das Marketing für öffentliche Güter von den Erfahrungen der Privatwirtschaft lernen? - = Arbeitsmaterialien zur Raumordnung und Raumplanung, Cahier 191, Lehrstuhl der Wirtschaftsgeographie und Regionalplanung der Universität Bayreuth, Bayreuth.

OFAT (1997): Le plan directeur cantonal. Guide de la planification directrice. - Directives en vertu de l'art 8 , OAT, Berne.

\section{Résumé: Le controlling dans la planification directrice cantonale}

La pratique actuelle de l'orientation de la planification implique un retoilettage complet de celle-ci à chaque décennie. Le degré d'efficacité de la planification directrice ne peut être évalué qu'approximativement avant l'évolution suivante, dans la mesure où celle-ci n'est entreprise que très peu de temps avant la révision générale. Dans beaucoup de cas un aperçu des développements ou effets, souhaités ou non, des mesures envisagées pour la réalisation des objectifs du plan, fait défaut. C'est pourquoi il n'est pas possible d'introduire des corrections dans l'évolution du développement spatial.

Une évolution fondée sur le principe du développement durable requiert toutefois de telles possibilités d'intervention. Il importe de mettre au point des démarches susceptibles de constater en temps utile les éventuelles déviations et de rectifier le cours de la planification.

Il existe des instruments qui permettent de surveiller et d'orienter la mise en œuvre des objectifs et mesures des plans cantonaux. Ils peuvent aussi contribuer à l'évaluation du degré de réalisation des objectifs définis par la planification. Il est ainsi possible de déceler en temps opportun les développements non durables et de procéder aux correctifs nécessaires. Ces instruments s'appellent monitoring, controlling et benchmarking; ils sont sous-tendus par des indicateurs. L'instrument central de ce système, le controlling, est présenté dans notre étude; son degré d'efficacité dans la planification cantonale directrice donne lieu à une analyse critique. Le recours au monitoring, au controlling et au benchmarking est indispensable pour un développement durable et une planification volontariste dynamique. 


\section{Zusammenfassung: Der Einsatz von Controlling in der kantonalen Richtplanung}

In der heutigen Praxis der Richtplanung findet im Allgemeinen alle zehn Jahre eine gesamthafte Überarbeitung des Richtplans statt. Die Wirksamkeit des Richtplans kann bis zur nächsten Evaluation, die meist erst knapp vor der Totalrevision durchgeführt wird, nur grob geschätzt werden. Eine Übersicht über erwünschte und unerwünschte Entwicklungen oder Wirkungen von Massnahmen des Richtplans fehlt in vielen Fällen. Damit kann auch nicht steuernd und korrigierend in die räumliche Entwicklung eingegriffen werden.

Eine am Prinzip der Nachhaltigkeit ausgerichtete Entwicklung bedarf aber gerade solcher Steuerungsmöglichkeiten. Es sind Vorkehrungen nötig, um Abweichungen vom gewählten Kurs erkennbar zu machen und allfällige Kurskorrekturen einzuleiten.

Es gibt Instrumente, welche die Umsetzung von Zielen und Massnahmen der kantonalen Richtpläne überwachen und steuern können. Sie können auch zur Bewertung beitragen, ob die erwünschten Entwicklungen eingetreten sind. Nicht nachhaltige räumliche Entwicklungen können so frühzeitig erkannt und mit geeigneten Massnahmen korrigiert werden. Solche Instrumente sind Monitoring, Controlling und Benchmarking (mit Hilfe von Indikatoren). Das zentrale Instrument dieses Systems, das Controlling, wird vorgestellt und seine Einsatzmöglichkeit in der kantonalen Richtplanung wird diskutiert. Für eine nachhaltige räumliche Entwicklung und eine «dynamische» Richtplanung ist der Einsatz von Monitoring, Controlling und Benchmarking unabdingbar.

\section{Summary: Controlling Tools in Swiss Cantonal Planning}

In Swiss cantonal planning today, the directive plan is reviewed generally every ten years. The plan is evaluated shortly before the next total revision, thus allowing only a rough estimate of its effectiveness. In many cases, a survey of the desired and unwanted spatial developments or effects of the plan's implementation is missing. It is therefore difficult to rectify or control undesired effects of spatial development.
Development that is geared to the principle of sustainability, however, needs exactly such possibilities of control. Precautionary measures are necessary to be able to identify course deviations and to allow for course corrections.

Tools are available which allow the supervision and control of goals and measures foreseen in the cantonal directive plan. They also enable an evaluation of the success of the desired development. Unsustainable spatial development can thus be timely recognised and rectified with appropriate measures. Tools useful for the above purposes are monitoring, controlling and benchmarking (with the support of indicators). The most important of these tools, controlling, is presented here and its possibilities of application in the cantonal directive planning are discussed. In order to achieve sustainable spatial development and a more dynamic directive planning, the implementation of monitoring, controlling and benchmarking is essential.

Dr. Marco Keiner, ORL-Institut, ETH Hönggerberg, CH-8093 Zurich.

e-mail:keiner@orl.arch.ethz.ch

Nicolas Mettan, C.E.A.T., 14 Avenue de l'EgliseAnglaise, CP 555, CH-1001 Lausanne.

e-mail: nicolas.mettan@epfl.ch

Barbara Schultz, ORL-Institut, ETH Hönggerberg, CH-8093 Zurich.

e-mail:schultz@orl.arch.ethz.ch

Manuskripteingang/received/manuscrit entré le 24.9.2001

Annahme zum Druck/accepted for publication/accepté pour l'impression: 23.5 .2002 